\title{
Alimentación, cultura y economía social. Los efectos de la crisis socioeconómica en la alimentación en Cataluña (España)
}

\author{
F. XAVIER MEdinA \\ Doctor en antropología social (Universidad de Barcelona) \\ Profesor investigador de la Universitat Oberta de Catalunya \\ Barcelona, España \\ fxmedina@gmail.com \\ Alicia AgUilar \\ Doctora en Biología (Universidad de Barcelona) \\ Profesora en la Universitat Oberta de Catalunya \\ Barcelona, España \\ aaguilarmart@uoc.edu \\ DAVID FORNONS \\ Doctor en Antropología Social y Cultural (Universidad de Barcelona) \\ Profesor en la Universitat Oberta de Catalunya \\ Barcelona, España
}

\begin{abstract}
Resumen En un periodo de crisis socioeconómica como el que vive actualmente Europa, y en especial los países del sur, el poder adquisitivo de la población y el acceso a los alimentos pueden verse fuertemente alterados. Dicha crisis, sin embargo, no afecta únicamente a los aspectos materiales de la alimentación y al consumo, sino que, por el contrario, implica también una dimensión sociocultural más amplia que afecta a los estilos de vida de modo más integral. El objetivo de este artículo es observar en qué medida la crisis socioeconómica que se inició en 2008 y que aún perdura, ha afectado a los hábitos de consumo y los estilos de vida de la comunidad autónoma de Cataluña (España). Para ello, se ha llevado a cabo en los últimos meses un trabajo de campo con entrevistas en diferentes estructuras familiares catalanas, de distintas características socioeconómicas, a través de las cuales los propios actores nos explican tanto su visión sobre cómo la crisis ha influido en su alimentación, como las estrategias que han puesto en marcha para hacer frente a esta transformación forzada de sus hábitos alimentarios cotidianos.
\end{abstract}

Palabras clave: alimentación, cultura alimentaria, crisis, economía, Cataluña, España, Europa

\section{Introducción necesaria}

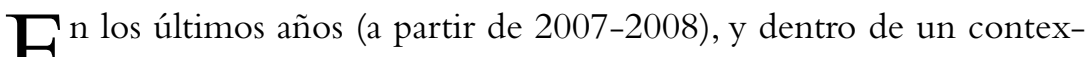
Eto de crisis mundial, determinados países de Europa como Grecia, Portugal, Italia, Irlanda ${ }^{1}$, Chipre o España, se han visto inmersos en una 
Figura 1 - Variación del consumo per cápita en Cataluña entre 2008 y 2012 (kg per cápita)

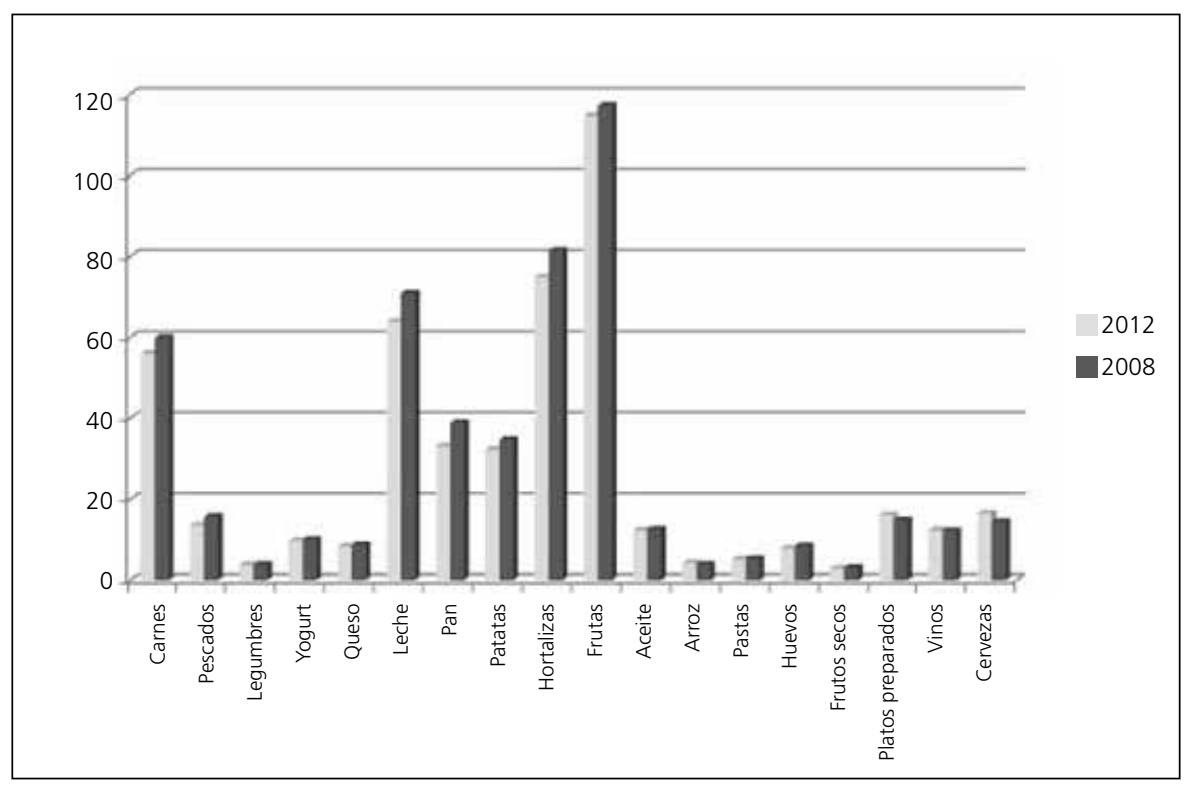

Fuente: elaboración propia en base a los datos del Panel de consumo alimentario del Ministerio de Agricultura Medioambiente y Alimentación (2012).

de las peores (si no la peor) crisis socioeconómica de su historia. En el caso de España, la crisis ha afectado (y aún afecta) directamente al empleo, con una importante destrucción del mismoª ; la banca, que ha necesitado un rescate europeo y grandes inversiones en una situación sin precedentes; $y$, por ende, al endeudamiento de las administraciones públicas ${ }^{3}$. Toda esta situación ha implicado un significativo empobrecimiento de la población (especialmente de sus capas más bajas y medias-bajas, que son las que más han recibido los efectos de la crisis) y del país en general, que ha afectado a todos los niveles.

La alimentación, en tanto que parte de las dinámicas socioculturales, está también sujeta a los fenómenos globales en los que las sociedades modernas actuales se encuentran inmersas. Así, en periodos de crisis socioeconómicas como el actual, y aunque el sector de la alimentación es uno de los que tradicionalmente resiste mejor las fluctuaciones de la economía, el acceso y la elección de los alimentos sufren alteraciones en mayor o menor grado.

El objetivo de este artículo es observar cómo la crisis socioeconómica ha afectado en los hábitos de consumo de la comunidad autónoma de Cataluña, en el Noreste del Estado español. Cataluña es considerada como el motor económico de España, y su producto interior bruto (PIB) anual es el más alto del Estado ${ }^{4}$. A través de entrevistas a profundidad en diferentes estructuras familiares de distintas características socioeconómicas de la comunidad autónoma de Cataluña, hemos intentado que los propios actores se adueñen del discurso y nos expliquen tanto su visión sobre cómo la crisis socioeconómica ha influido en su alimentación, como las estrategias que han puesto en marcha para hacer frente a esta transformación forzada de sus hábitos alimentarios cotidianos.

\section{Comer en tiempos de crisis}

Las pautas de consumo, de acuerdo con la denominada Ley de Engel (cf. Cáceres et al., 2013) tienden a presentar el siguiente comportamiento en situaciones de crisis económica: a medida que tiene lugar un aumento de los ingresos, disminuye la proporción de gasto dedicada a la satisfacción de las necesidades básicas (alimentación, vestuario, vivienda, etc.), mientras que aumenta en porcentaje aquella destinada a aquellos bienes no básicos. En consecuencia, en un contexto de crisis y de disminución de los ingresos, se espera que dicha ley se cumpla de forma inversa, aumentando el porcentaje de gasto en productos básicos y disminuyendo aquel que se refiere a productos superfluos.

Hace relativamente pocos años, en el momento inicial de la crisis socioeconómica en España, autores como García Álvarez-Coque y López-García (2009)

2 Aunque desde el segundo trimestre de 2014 los datos del paro en España empiezan a mostrar una muy tímida recuperación, la tasa de paro en España en junio de 2014 es del 24,5\% (http://www.rankia.com/blog/tasa-paro/2095409-evolucion-tasa-paro-2014).

3 En el segundo trimestre de 2014, la deuda alcanzó ya el 98,40\% del PIB en España y supera el billón de euros (http://www.datosmacro.com/ deuda/espana).

4 http://www.datosmacro.com/pib/espana-comunidades-autonomas 
Tabla I - Variación del consumo per cápita en Cataluña entre los años 2008 y 2012

\begin{tabular}{|c|c|c|c|c|c|}
\hline \multicolumn{6}{|c|}{ Producto } \\
\hline \multicolumn{2}{|c|}{ Carnes } & \multicolumn{2}{|c|}{ Pescados } & \multicolumn{2}{|c|}{ Leche } \\
\hline Vacuno & $-2,01$ & Frescos & $-2,01$ & Entera & $-5,51$ \\
\hline Pollo & $-0,17$ & Congelados & $-0,17$ & Desnatada & $-0,36$ \\
\hline Cerdo & $-0,64$ & Mariscos & $-1,68$ & Semi & $-1,57$ \\
\hline Conejo & $-0,33$ & Sardina & $-0,54$ & & \\
\hline Ovino/caprino & $-0,91$ & Rape & $-0,59$ & & \\
\hline Pavo & $+0,12$ & & & & \\
\hline Jamón & $-0,13$ & & & & \\
\hline Jamón ibérico & $-0,05$ & & & & \\
\hline \multicolumn{2}{|c|}{ Hortalizas y verduras } & \multicolumn{2}{|c|}{ Frutas } & \multicolumn{2}{|c|}{ Otros } \\
\hline Frescas & $-6,56$ & Frescas & $-2,54$ & Plat. preparados & $+1,2$ \\
\hline Congeladas & $-0,2$ & Congeladas & -0.2 & Cerveza & $+1,96$ \\
\hline Ecológicas & $+1,05$ & Ecológicas & $+1,19$ & Vinos & $+0,13$ \\
\hline IV gama & $-0,09$ & IV gama & $-0,24$ & & \\
\hline
\end{tabular}

Fuente: elaboración propia en base a los datos del Panel de consumo alimentario del Ministerio de Agricultura Medioambiente y Alimentación (2012).

apuntaban ya el hecho de que en momentos de recesión, el consumidor responde comprando menos alimentos y, especialmente, aquellos que resultan más baratos. Por lo tanto, y como parece evidente, aspectos tales como el aumento de los niveles generales de precios de los alimentos o la disminución del poder adquisitivo de la población afectan directamente a los patrones de consumo. Según estos autores, cuando el presupuesto disponible para alimentación es reducido, la estrategia económica más racional suele ser maximizar la ingesta de calorías, por lo que el consumidor se orienta hacia alimentos más baratos, que acostumbran a tener más grasa y por tanto más energía, al mismo tiempo que proporcionan una mayor sensación de saciedad. Es lo que Patricia Aguirre (2005: 167 y ss.) dio en llamar en su día para el caso de Argentina "alimentos rendidores", que son aquellos que, consumidos por la población con mayores dificultades socioeconómicas, cumplen con tres requisitos: deben ser baratos, deben llenar y deben gustar.

Estos alimentos suelen ser procesados. Por consiguiente, los productos frescos como las frutas y las hortalizas son, junto con la carne y los productos lácteos, aquellos que más afectados se ven cuando se reduce el poder de compra de los consumidores.

En este mismo sentido, según datos del Ministerio de Agricultura, Alimentación y Medio Ambiente, por primera vez desde 2004, el precio es el principal factor a la hora de decidir comprar en un establecimiento, y el 62,7\% de los encuestados para el Panel de consumo alimentario de España en 2012 afirmaban que se deciden por los mejores precios, aparte de las ofertas ocasionales.
Se constata también una reducción de un $12 \%$ del consumo extradoméstico de alimentos desde 2009.

Si nos remitimos a los datos para Cataluña del consumo per cápita en 2008, año en que podríamos situar prácticamente el inicio de la crisis, y se comparan con los de 2012, se ratifica esta tendencia a la baja en el consumo de alimentos en general $(746,87 \mathrm{~kg}$ de alimentos per cápita en 2008 frente a 705,62 kg en 2012), con mayor afectación para el grupo de la leche (no así para los yogures y quesos, que bajan de manera moderada), las hortalizas y el pan, seguidos de la carne, las frutas, las patatas y el pescado. En sentido contrario, se observa un ligero aumento en el consumo de cerveza y de platos preparados (Figura 1).

En un análisis un poco más detallado de estos grupos alimentarios se observa cómo, efectivamente, la caída de productos con precios medios más caros es superior en algunos de los grupos, como en el de las carnes o los pescados, donde el vacuno, el marisco y los pescados frescos, con precio superior en el mismo grupo, son los que parecen acusar más la crisis.

Sin embargo, observamos también que algunos productos con ciertas particularidades y con precios más elevados dentro de su mismo grupo alimentario, como las verduras y hortalizas ecológicas, la leche desnatada frente a la entera o el incremento en el consumo de platos preparados, ponen en evidencia la multiplicidad de factores que llevan al consumidor a decantarse por la adquisición de unos u otros productos y cómo el mercado evoluciona para satisfacer las nuevas demandas -platos preparados, productos de cuarta gama, alimentos funcionales. (Sánchez, 2008). 
La preocupación por la salud, la falta de tiempo, la predisposición hacia el consumo de productos específicos y locales, vinculados con la cultura y la identidad (Contreras y Gracia, 2005) es un hecho social que está sujeto también a los fenómenos y transformaciones sociales como el que estamos investigando aquí: la crisis económica y su vinculación a la alimentación.

Observamos asimismo, tanto en los estudios cuantitativos sobre el tema como en el nuestro, cualitativo, en particular, cómo la preocupación por la salud y la alimentación sigue siendo un eje primordial en nuestra sociedad. Probablemente, en este aspecto cabría quizás hablar de la adecuación del "comer sano" a la crisis más que un cambio en el concepto y la práctica del "comer sano".

Así pues, al margen de la mera estimación material sobre los efectos de la crisis en la cesta de la compra, el objetivo de este trabajo es determinar si los cambios alimentarios que implica la crisis socioeconómica conllevan también una dimensión sociocultural más amplia que afecta a los estilos de vida de modo más integral.

\section{Metodología}

Para dar respuesta a los interrogantes principales de la investigación se llevó a cabo un estudio de carácter cualitativo mediante entrevistas semidirigidas a consumidores de estructuras familiares y socioeconómicas diferentes de la comunidad autónoma de Cataluña. Dicho trabajo de campo se desarrolló entre los meses de septiembre de 2012 y junio de 2013 -periodo que, a nuestro entender, puede considerarse como relativamente intenso dentro del panorama del desarrollo de la crisis socioeconómica española-.

Se preguntó sobre cómo la crisis estaba afectando a sus hábitos y prácticas alimentarias a treinta personas de ambos sexos (19 mujeres y 11 hombres) con edades comprendidas entre los 21 y 55 años, aunque la franja más representada en la muestra es aquella que va de los 30 a los 45 años. Es difícil, sin embargo, determinar el estatus socioeconómico exacto de la muestra. En el acceso a los integrantes de la muestra ha tenido que ver tanto el contexto como las posibilidades de acceso y los contactos de los distintos investigadores que han llevado a cabo el trabajo de campo; en este sentido, se ha intentado, de manera subjetiva, buscar informantes pertenecientes a unidades familiares que, en relación a sus ingresos, pertenezcan a lo que se ha dado en definir como clase media y clase media baja (aquellos que ingresan menos de 60.000 euros anuales).

Las entrevistas se llevaron a cabo en catalán y en castellano, dependiendo de la lengua habitual de uso del o de la informante. Para su inclusión en este trabajo, aquellos fragmentos de discurso obtenidos en lengua catalana han sido traducidos por los mismos autores de este artículo. Cinco de los informantes (cuatro mujeres y un hombre) eran oriundos de otros países, aunque siempre con suficiente dominio de alguna de las dos lenguas utilizadas en el estudio.

Durante la entrevista se recabó información del nivel de ingresos de la unidad familiar, sobre el número de miembros del grupo familiar directo que dependían directamente del informante en temas de alimentación, y sobre qué comían antes de la crisis y qué comían actualmente. Se les pidió que reflexionaran sobre los cambios, si los había habido o no y cuáles, en relación con su alimentación, no sólo sobre los aspectos materiales, sino también nutricionales y sociales; también si conocían casos de familiares, amigos o conocidos afectados por la crisis en sus hábitos alimentarios.

\section{Impresiones y actitudes ante la crisis}

La totalidad de los entrevistados piensan que, en mayor o menor medida, la crisis socioeconómica les ha afectado, tanto a ellos como -especialmente- a su entorno. Igualmente, y también más en unos casos que en otros, la totalidad de los encuestados afirman haber variado sus hábitos de consumo, aun mínimamente, por causa de la crisis: "No hemos cambiado mucho la compra, pero algunas cosas sí, claro... (...) Es inevitable” (Sara, 39 años).

En función del género, el más preocupado y, por ende, el que se considera más afectado es el femenino. Así, por ejemplo, las entrevistas más en profundidad, con un mayor nivel de reflexión y con más aportaciones fueron casi siempre las realizadas por las mujeres. Algunos hombres se refirieron a suposiciones y expresaron que "este tema" lo llevaban más sus compañeras, con la salvedad de los hombres que vivían solos o con otros hombres.

Por otro lado, las entrevistas han mostrado una mayor preocupación por parte de las mujeres hacia aquellos temas del consumo alimentario más relacionados con la salud. Así, aparece que aquellas personas afectadas por la crisis que tenían unos hábitos alimentarios subjetivamente considerados como saludables antes de la misma, están intentando mantenerlos a pesar de aspectos tales como la pérdida del trabajo, la reducción del poder adquisitivo o la posible presión psicológica derivada de la crisis.

Según la opinión de los informantes de esta investigación, se asocia, sin embargo, el comer saludable a aspectos diversos, que pueden ir desde preocupaciones de carácter médico nutricional ("mantener a raya el colesterol", "reducir los triglicéridos", etc.) o la preocupación por el peso o por mantener la línea, hasta el hecho de comer alimentos o cocina tradicional y al consumo de productos locales. Como señala Igor de Garine (s.f.: 28-29): "En cada consumidor coexisten tendencias a satisfacer su gula, tener una dieta sana, demostrar su nivel 
socioeconómico, su modernidad, al mismo tiempo que su autenticidad cultural".

Lo tradicional, lo "local", lo "casero" se valora especialmente. En palabras de una entrevistada: "Cocinar lo de nuestras madres, que es más sano y además más barato" (Marina, 43 años). La cocina tradicional parece volver a considerarse como un refugio seguro en tiempos de crisis, saludable y a buen precio. Como nos dice la misma informante: "Nuestras madres hacían guisos muy buenos, y ahorraban. Y eran más sanos y tenían menos grasas. Hay que recuperar las recetas tradicionales". Sin embargo, dicho retorno a lo tradicional parece estar en cierta medida determinado también por el entorno: "En el pueblo es más fácil volver a lo de siempre y a comer de la huerta" (Sonia, 37 años).

Sandra es vegana y se dedica a la naturopatía. Ella nos cuenta que a pesar de la crisis y de la disminución de los ingresos no renuncia a sus principios:

\begin{abstract}
“(...) La gente, y yo misma, no renuncia a sus principios. Nos privaremos de objetos más de lujo o de fiestas, pero la dieta base creo que pocos la han variado, a no ser que no tengan o no sepan donde recortar. La crisis pasará o no, pero una vez estás acostumbrada a comer sano y todo lo que significa, no lo cambias..., es una forma de vida y cada vez hay más gente e implica más que la dieta y lo que comemos, implica el entorno, todo (...)" (Sandra, 33 años).
\end{abstract}

Marina nuevamente, barcelonesa de 43 años, se niega a renunciar a una "comida saludable para mis hijos". Productos y marcas de primera línea serán para ellos, mientras que marcas blancas y productos de menor calidad y/o precio son para el resto de la familia: "Nosotros (padres) ya nos apañaremos y ya cambiarán las tornas". Vemos en este caso como se da una cierta relación a nivel de asociación mental entre precio, marca y calidad. Se considera que los productos de marca, que son más caros, son también mejores, mientras que los de las marcas blancas, más baratos, pierden calidad junto con la disminución de precio... Este mismo argumento lo encontramos en el discurso de Pau (barcelonés, 34 años), quien comenta que "(las marcas blancas) si son más baratas, será por algo. De algún sitio lo tienen que ahorrar". Sin embargo "la relación calidad precio que ofrecen es (suficiente) y muchas veces salen a cuenta".

En el análisis de las entrevistas se concluye que la preocupación por comer bien, consumir alimentos ecológicos (que, por cierto, continúan mostrando un consumo ascendente a pesar de la crisis) y seguir dietas y disposiciones saludables en la población sigue siendo importante, tanto en la forma de pensar como en la forma de comprar y consumir. Sin embargo, el problema de la asociación de la alimentación con la crisis se visibiliza cuando existe un déficit en conocimiento nutricional. Este déficit, junto con la disminución del poder adquisitivo, lleva a algunos de los informantes a elegir como primera premisa alimentos económicos, no todos ellos considerados por ellos mismos como saludables; a simplificar la variedad alimenticia (por ejemplo, primar la ingesta de proteínas con embutidos); y la alimentación fuera del hogar, de carácter más lúdico, realizarla por motivos de coste en establecimientos de menor precio (Fast Food, en ocasiones) ante la imposibilidad de poder acceder a otros establecimientos de restauración. Nuevamente Pau (barcelonés, 34 años) nos comenta que "Muchos restaurantes han bajado precios y han puesto menús más baratos, hasta en los días festivos. Seguramente (...) la calidad no es la misma, o la cantidad, pero para comer fuera están muy bien. (...) Creo que el consumidor ha salido ganando en parte con esto".

Marisa, colombiana de 39 años afincada en España desde 2003 y cuidadora de niños, afirma que cuando llegó:

\begin{abstract}
"Sí que poco a poco cambié mis hábitos de lo que comía, y ahora con la crisis sigo igual que antes de la crisis (...) Pero mi marido trabaja haciendo obras como ayudante de otro; cuando había más trabajo y cobraba más, comía al mediodía de menú en el bar, ahora, como no le gusta llevarse la comida, no la puede calentar, puede sentarse tranquilo, lo que hacen es irse a un McDonald's, donde pueden sentarse y la comida es más barata; casi con un poco más de tres euros tiene dos pequeñas hamburguesas y la cerveza (...) El menú de bar es de diez el más barato y depende del barrio en el que hacen la obra, con dos platos, bebida, postre o café (...)".
\end{abstract}

Graciela, ecuatoriana de 25 años y residente en Santa Coloma de Gramenet (Barcelona), nos dice que:

"Desde que estoy en España he engordado porque la alimentación aquí tiene más calidad y cantidad (...) Me gusta mucho la pasta y la comida italiana está bien de precio, el problema no son estos alimentos, son las cantidades y claro, es mejor si lo cocinas tu, si tienes tiempo para hacerlo. Aquí se come mucho bocadillo, pero es por el trabajo, si la gente tuviera más tiempo no comería tantos bocadillos".

A pesar de lo manifestado por Graciela, los entrevistados establecen una separación más clara entre alimentos básicos y secundarios, entendiendo los secundarios como no imprescindibles para la alimentación diaria y considerados más como placer o distintivo de clase.

Toni, de 47 años y en paro desde hace siete meses, trabajaba de comercial de impermeabilizaciones de suelos industriales:

"Sí, he cambiado de alimentación (...) He dejado lo que ahora considero como productos de lujo, como aceitunas, berberechos, aperitivos, Martini, jamón ibérico, filete de ternera buena..." 
Gloria, de 50 años, dependienta en una zapatería en Barcelona, dice asimismo que:

"Sí, he tenido que hacer muchos cambios. Mi sueldo ha disminuido y las horas que trabajo han aumentado, aunque mi padre nos ayuda (...) Principalmente hemos dejado de comer marisco y ternera..., y ya no me compro productos para adelgazar y de farmacia (...)".

Josep, estudiante en Barcelona, comparte el piso con otros tres chicos de su edad y de su mismo pueblo, todos entre 20 y 24 años:

"La crisis sí que nos afecta (...) Hemos clasificado entre alimentos necesarios y alimentos prescindibles. Compartimos un mínimo de alimentos en la nevera que compramos entre todos. Antes había productos menos necesarios, cervezas, otras bebidas o por ejemplo el jamón era de marca... Ahora tiramos de un mínimo para todos, y el que quiere y puede se lo compra aparte. Todos hemos reducido en el fondo el nivel y la calidad de lo que compramos, tanto si se puede como si no".

María, con dos hijos y con su marido desempleado desde hace tres años, nos dice:

"Si, he eliminado los embutidos, lo enlatado, el pan de caja (sic.) de marca, carne, pescado fuera de temporada, ropa y calzado, artículos de belleza.Y compro lo más barato, sobre todo los artículos de limpieza. En la comida procuro que sea mejor, aunque sea menos que antes".

Podemos destacar, a través del discurso de los informantes, que la alimentación, aun siendo una parte muy importante del consumo familiar, no se observa ni se valora al mismo nivel que el consumo de otros bienes y servicios. La comida, a raíz de lo manifestado por los informantes, es lo último a recortar en el presupuesto familiar; y especialmente, aquella que consumen los más pequeños. Helena, administrativa de 39 años y madre de dos hijas, nos dice en este sentido que:

"En lo último que se recorta es en la comida y sobre todo de las niñas (...) Si acaso nos privamos nosotros de caprichos y para nosotros compramos marca blanca, pero mientras se pueda será lo último en lo de las niñas. Ropa, zapatos, otras cosas, vale (...)".

En relación con las variaciones en los hábitos de consumo, observamos también que los informantes afirman no haber variado su lugar de compra, especialmente en los espacios urbanos. Son los productos los que afirman que han sido cambiados, tanto a nivel de marca como de cantidad, en algunos casos: "No, el (establecimiento) en el que compro es bueno y tiene buenos precios. Sigo comprando ahí, pero sí que miras más lo que gastas, claro" (Pau, barcelonés, 34 años),
En cambio, en los entornos rurales sí que parece haberse producido un cierto fenómeno de cambio del lugar de compra, cambiando el consumo más cercano, el comercio local y próximo, por grandes superficies a las afueras de la localidad o en alguna localidad próxima. En este sentido, manifiestan haber cambiado también los productos consumidos, incrementando ciertos productos: carnes, pollo, bollería..., adquiridos en las grandes superficies, en detrimento de los productos de la carnicería o panadería locales. Se afirma haber aumentado también el consumo de marcas blancas.

Oliver, de 42 años, con padres oriundos de Francia y afincado en un pueblo de la provincia de Girona, cerca de la frontera con Francia, nos dice:

"Solía ir a comprar a Perpignan cada 15 días al (nombre del supermercado), queso, foie, jamón de pato, chocolates... Ahora, en espera de volver a tener trabajo, no puedo permitírmelo por el precio de la gasolina y de los productos que solía consumir (...) Volvemos a vivir con mis padres (...)".

Clara, de 43 años, que vive en Sant Quirze de Besora (a pocos kilómetros de Ripoll, en la provincia de Girona), afirma que: "Ahora compro más en Ripoll en (nombre de una im-
portante cadena local de supermercados), porque no
tengo más remedio, aquí (en su localidad de residen-
cia) dos de los tres hornos (panaderías) han cerrado y
la ternera me cuesta casi la mitad (en el supermercado),
aunque ya sé que es peor (...)".

Toni, de 47 años, en paro desde hace siete meses:

"También he cambiado donde compro, he dejado de comprar en las tiendas del pueblo; bueno, en las que menos relación tenía, y compro en el supermercado. Por ejemplo, ahora compro el pan en la gasolinera y con un euro tengo dos baguettes...".

Observamos, asimismo, cómo los cambios que la crisis está produciendo en los entrevistados van más allá del cambio de productos, precios y lugares en los cuales adquieren los alimentos, sino que afecta a sus hábitos de vida en general, incluyendo las relaciones que a nivel de comercio -y por tanto de relación vecinal- se daban en el mundo local; y, muy particularmente, a aquellos aspectos de la cotidianidad más relacionados con el ocio. Como atestigua Gloria, de 50 años y dependienta en Barcelona:

“Antes salíamos a comer fuera los domingos. Ahora es mi padre quien cada quince días paga la comida, y ahora, aunque sea tarde, comemos siempre en casa y cenamos (...) Las marcas que compro, antes ni lo miraba, ahora compro en (cita dos nombres de supermercados 
de precios muy económicos), intentando no comprar marcas conocidas, no quiero pagar la marca (...)"

Pero si bien los informantes hablan sobre la crisis y cómo ésta les afecta en su alimentación, se produce una extensión, vemos también que se crea una cierta empatía sobre la situación en general de todo el conjunto, más allá del círculo social y familiar más cercano.

"Yo no he sentido mucho la crisis, pero conozco gente que sí. Nosotros, por suerte, estamos bien y nos podemos permitir lo que sea (...) Pero igualmente miramos más las cosas, claro. Hay gente que lo está pasando mal y no están las cosas como para dispendios (...)" (Carles, 47 años).

"Antes tiraba más cosas. Ahora las aprovecho. Me da pena tirar comida, cuando hay tanta gente que la necesita" (Sara, 39 años).

“A nosotros no nos ha afectado mucho. Bueno, quizás lo notas a nivel general... Pero a mucha gente le ha tocado de lleno (...)" (Pau, 34 años),

Esta empatía, este pensar en el "otro" como sujeto afectado por la crisis, implica una cierta sensación de "corresponsabilidad" con el otro y con la sociedad en general, incluso provocando cambios en la concepción de la propia alimentación. Es decir, que sin sufrir cambios o privaciones específicos en determinados artículos y prácticas alimentarías, los informantes establecen nuevas consideraciones y valoraciones sobre la alimentación, entre ellas la de un rechazo de cierto esnobismo alimentario o cierta exhibición de recursos. Se afirma que se "aprovecha" ${ }^{5}$ " y se recicla más, por ejemplo. Una solidaridad hacia el otro, afectado, que establece, quizás, en algunos niveles, un cierto cambio de valores, de significados sobre la comida y la alimentación, que se refleja incluso en un cambio de hábitos y de costumbres.

Isabel, de 35 años, trabaja en una autoescuela:

\footnotetext{
"Ahora la autoescuela no va tan bien, hay menos gente que se saca el carné de conducir, pero no me ha afectado, aunque me he vuelto más ahorradora. Antes compraba muchos productos macrobióticos y ecológicos, ahora ya no. También lo que pasa es que muchas amigas y amigos míos lo están pasando francamente mal con la crisis, así cuando quedamos reducimos gastos, me adapto, porque podría haberme pasado a mí (...)".
}

\begin{abstract}
“Tengo un hijo de 9 años y una hija de 20. El niño come en casa. Antes, alguna vez se quedaba a comer en el comedor de la escuela, como premio para jugar con sus amigos que se quedan cada día (...) Mi hija es universitaria y antes se quedaba a comer en el bar de la facultad, que es muy económico, pero ahora se lleva la fiambrera de casa, ya que sus amigas lo hacen, un poco por solidaridad y para comer con ellas" (María, 51 años).
\end{abstract}

Olga, mujer de 67 años, jubilada, de Tarragona, nos comenta también que:

"Nuestra situación no ha variado, nuestros ingresos casi no los han tocado. (...) Somos muy metódicos y la verdad es que hacemos exactamente lo mismo que hacíamos, comemos lo mismo. Ahora bien, sí que vemos en nuestro alrededor que hay gente joven que lo está pasando mal, con vecinos que alguno de los dos (del matrimonio) ha perdido el trabajo (...)".

"Por suerte mi entidad no está en peligro ni va ha hacer regulaciones a corto plazo, pero a familiares y amigos sí que le está afectando y mucho. Por ejemplo mi hermano, arquitecto, y ahora solo trabaja por proyectos, no de forma continua (...) Lo que no me parece bien es mantener ciertos hábitos y caprichos y con la que está cayendo seguir igual. Al fin y al cabo es como el refrán, cuando veas las barbas de tu vecino cortar, pon las tuyas a remojar. Incluso en las comidas de la entidad se ha reducido el gasto, y no sólo por imagen; en el fondo no estaba bien tanto dispendio, sobre todo en la comida de Navidad. En casa quizá (nombre de su mujer) se ha concienciado más a comer sano y tal, pero puertas adentro no hemos cambiado" (Pere, director de banco, 43 años).

\section{De crisis, consumo y cambio de hábitos alimentarios}

Es evidente que la crisis no afecta por igual a todo el mundo. Sin embargo, la contracción del consumo (¿deberíamos hablar aquí de consumismo?) empieza a ser notable. Los cambios en los hábitos alimentarios responden a distintas causas, y distan mucho de ofrecer una visión unívoca o simplista de la vivencia que los individuos manifiestan en este tiempo de crisis. Sin embargo, sí que podemos establecer determinadas líneas 
conclusivas que parecen desprenderse del discurso de nuestros propios entrevistados.

En primer lugar, no parece discutirse la pérdida generalizada de capacidad adquisitiva de la población (desempleados, expedientes de regulación de empleo en las empresas, recortes en los sueldos...). En segundo lugar, la situación parece crear en todos los entrevistados un cierto efecto psicológico negativo debido a la situación social de inseguridad generalizada -que se alimenta y actualiza a través de los medios de comunicación- y que lleva a adoptar un estilo de vida más austero, y tanto si se sienten directamente afectados por la crisis como si no. Esta sensación de inseguridad constatada, lleva los individuos a ser en ocasiones más conscientes socialmente (algunos informantes manifiestan comprar ahora, cuando se puede, en la granja, en las cooperativas de consumo, productos ecológicos, etc.; o tiran menos y reaprovechan más las sobras), más solidarios (ayudar a quien lo necesita, aunque más a nivel genérico, como donar alimentos a ONG o a asociaciones, que directamente a personas concretas fuera del ámbito familiar). También a nivel corporativo, aunque la empresa no esté afectada por la crisis, los "dispendios" de las cenas de navidades o de empresa se han reducido. La misma administración autonómica (Generalitat de Catalunya) ha retirado prácticamente toda subvención de los menús y de los vales de comida para sus empleados.

Las entrevistas parecen confirmar, en términos generales, el descenso en el consumo de determinados alimentos que muestran los datos estadísticos (Panel... 2012). Desciende el consumo de frutas, hortalizas, pescados frescos, mariscos y carnes, y en ello podemos vislumbrar quizás una posible correlación con el precio de los mismos. Sin embargo, vemos que, precisamente, son las frutas y verduras ecológicas aquellas que presentan un aumento más significativo. En este caso, podemos encontrar dos líneas argumentales que explicarían este hecho: por un lado, el auge de lo ecológico corre paralelo a la crisis, situándose en su interior, pero con un discurso propio. Por otro lado, posiblemente el aumento de frutas y verduras ecológicas hayan sustituido en alguna medida el consumo de las no ecológicas, formando parte, de este modo, de la explicación del descenso de su consumo.

Sin embargo, observamos también un descenso de otros alimentos mucho más baratos y, al menos en teoría, base de la alimentación cotidiana, como el pan y las legumbres. En este caso, no podemos aducir problemas de precio, sino de otro tipo (¿calorías, tiempo...?). En la parte contraria, encontramos un aumento notable del consumo de vinos y cervezas, que son productos que sólo marginalmente pueden ser considerados como de primera necesidad. Dentro de este grupo, en el año 2011, los vinos con Denominación de Origen aumentaron su consumo mientras que descendía el de los vinos de mesa de menor calidad (Consumo de vino... 2011).
Finalmente, no hemos podido conseguir confirmación alguna entre los entrevistados que explique el significativo descenso del consumo de leche que muestran las estadísticas ( $c f$. Figura 1, Tabla 1), siendo además el único lácteo que se desploma claramente, mientras que quesos y yogures mantienen sus posiciones, quizás sustentados en la amplia gama y variedad existente que ha hecho crecer el mercado en los últimos años (Collantes, 2012).

Observamos que la situación personal es determinante en la percepción general de la situación, pero no es la única. Somos capaces de percibir la situación económica de modo abstracto, sí, pero la propia experiencia vital, nuestra propia situación y la de nuestro entorno marca sensiblemente nuestra experiencia de la crisis. Como humanos, ejemplificamos lo abstracto tanto con lo que nos ha sucedido directamente como con los casos reales que conocemos. Sin embargo, vemos también que el panorama general y los casos concretos que vamos conociendo a través de los medios de comunicación marcan también nuestra percepción global de la situación, y crean un clima de crisis compartida, incluso entre aquellos que afirman no padecerla directamente.

Es destacable, por otro lado, en apariencia al menos, la existencia de una cierta resignación por parte de los entrevistados ante una situación que nos supera y que es, cuando menos, dificil de aprehender. Hay algún informante que manifiesta que "antes" se vivía por encima de las posibilidades y una manifestación era el consumo de alimentos más caros y en ocasiones "esnobs" e innecesarios, y en ocasiones "estirando más el brazo que la manga".

Finalmente, parece existir en algunos casos una mayor percepción del cambio llevado a cabo que la incidencia real del cambio en si mismo. Los informantes plantean en la totalidad de los casos haberse sentido víctimas de la crisis en mayor o menor medida, e incluso haber modificado sus compras y sus hábitos alimentarios; sin embargo, algunos de ellos -aquellos que están en una situación económica más desahogada- especifican posteriormente que los cambios llevados a cabo en realidad no han sido tales o bien han sido mínimos, o bien que se han visto inducidos a ellos de manera más indirecta (situación de los amigos, recortes en gasto de la empresa) que directa.

A través de las distintas entrevistas llevadas a cabo, hemos intentado conceder voz a los propios actores implicados en la crisis, quienes se adueñan del discurso y nos dan su propia versión, desde dentro, tanto sobre cómo la crisis socioeconómica ha influido en su alimentación, como de las distintas estrategias puestas en marcha para hacer frente a esta transformación forzada (o no) de sus hábitos alimentarios cotidianos.

El acceso a los alimentos y la posibilidad de su consumo en función de la situación socioeconómica de las unidades familiares impone a los actores la necesidad de poner en práctica nuevas estrategias adaptativas 
de funcionamiento tanto doméstico como social. Sin embargo, y dentro de un abanico controlado de posibilidades, el rubro alimentario es habitualmente uno de los que se ve afectado en último lugar.

Las crisis, en tanto que épocas de cambio, y en cualquier momento de la historia, han servido para pensar y para poner a prueba nuevas estrategias sociales y culturales; y la presente crisis, a pesar de no haber terminado todavía, no es en absoluto una excepción.

\section{Referencias}

AGUIRRE, Patricia. Estrategias de consumo. Qué comen los argentinos que comen. Buenos Aires: CIEPP-Miño y Dávila, 2005. CÁCERES, Juanjo; MEDINA. F. Xavier; ESPEITX, Elena y CANTARERO, Luis. Nuevas tecnologias alimentarias: aceptabilidad y rechazo social en tiempos de crisis. In: González Turmo, I. (ed.) Respuestas alimentarias a la crisis económica. Sevilla: International Commission on the Anthropology of Food and Nutrition (ICAF), 2013.

COLLANTES, Fernando. El consumo de productos lácteos en España, 1950-2010. In: III Encuentro Anual de la Asociación Española de Historia Económica. Sociedad Española de Historia Agraria (Documentos de Trabajo), 2012. http://www. ub.edu/histeco/3encuentro/pdf/collantes.pdf (consultado el 15 de julio de 2013).
COLlinSON, Paul. Food and the Economic Crisis in Ireland. In: González Turmo, I. (ed.) Respuestas alimentarias a la crisis económica. Sevilla: International Commission on the Anthropology of Food and Nutrition (ICAF), 2013.

CONTRERAS, Jesús y Gracia, Mabel. Alimentación y cultura. Perspectivas antropológicas. Barcelona: Ariel, 2005.

Consumo de vino en hogares en España. Madrid: Observatorio Español del Mercado delVino, 2011.

GARINE, Igor de. Antropología de la alimentación: entre naturaleza y cultura. Valencia: Museu Valencià l'Etnologia, sd. Disponible en: http://www.museuvalenciaetnologia.es/userfiles/file/Ernaehrung_und_Kultur.pdf (consultado el 18 de junio de 2013). GARCÍA ÁLVAREZ-COQUE, José M. y LÓPEZ-GARCÍA, Teresa. Los cambios en el consumo alimentario. Repercusiones en los productos mediterráneos. In: Lamo Espinosa, J. (coord.), El nuevo sistema agroalimentario en una crisis global. Almería: Cajamar, 2009. Disponible en: http://www. publicacionescajamar.es/pdf/publicaciones-periodicas/mediterraneo-economico/15/15-262.pdf (consultado el 12 de julio de 2013).

Panel de consumo alimentario 2008 a 2012 (diversas series). Madrid: Ministerio de Agricultura Medioambiente y Alimentación, 2013. Disponible en: http://www.magrama.gob.es/ es/alimentacion/temas/consumo-y-comercializacion-y-distribucion-alimentaria/Revisado_1300307_PANEL_CONSUMO_vFinal_NPD_tcm7-270351.pdf (consultado el 27 junio de 2013).

SÁNCHEZ, Mercedes. Los nuevos consumidores. In: Papeles de economía española, 117, 2008, pp. 157-166.

\title{
Food, culture and social economy. The effects of socio-economic crisis in Food in Catalonia (Spain)
}

\begin{abstract}
In a period of social and economic crisis like the current one in Europe, and especially in the southern countries, the purchasing power of the population and their access to food can be strongly altered. This crisis, however, is not confined only to the material aspects of food and consumption, but on the other hand, it also implies a broader sociocultural dimension that affects the lifestyles in a bigger comprehensive way. The aim of this paper is to observe how this socio-economic crisis that began in 2008, has affected the consumer habits and lifestyles of the population living in Catalonia (Spain). This has been carried out recently in a fieldwork, interviewing members of different Catalan family structures of different socioeconomic characteristics, through which the actors tell us their views on both how the crisis has affected their food and the strategies that have been implemented to address this forced transformation of their daily food habits.
\end{abstract}

Keywords Food, food culture, crisis, economy, Catalonia, Spain, Europe

\section{Comida, cultura e economia social. Os efeitos da crise socioeconómica na alimentação, na Catalunha (Espanha)}

\section{Resumo}

Em um período de crise econômica e social como a que é vivida atualmente na Europa de hoje, especialmente nos países do sul, o poder de compra e o acesso aos alimentos da população podem ser fortemente alterados. Esta crise, no entanto, não se limita aos aspectos materiais dos 
alimentos e do consumo, mas, por outro lado, também implica uma dimensão sociocultural mais ampla que afeta o estilo de vida de forma mais abrangente. O objetivo deste trabalho é observar até que ponto a crise socioeconômica que começou em 2008 e que ainda continua, tem afetado os hábitos de consumo e estilos de vida da região autônoma da Catalunha (Espanha). O trabalho de campo foi realizado com entrevistas a membros de famlías catalãs diferentemente estruturadas e de diferentes características socioeconômicas. Através das entrevistas os atores nos contam suas opiniões sobre como a crise tem afetado sua comida e as estratégias que foram implementadas para lidar com essa transformação forçada de seus hábitos alimentares diários.

Palavras-chave Comida, cultura alimentar, crise, economia, Catalunha, Espanha, Europa

Data de recebimento: 15-03-2015

Data de aceitação: 30-09-2015 